\title{
La investigación como estrategia para fomentar valores entre estudiantes de noveno grado de la institución educativa departamental rural de Cantagallar apoyada en las TIC ${ }^{1}$ \\ Research as a strategy to promote values among ninth-grade students of the rural departmental educational institution of Cantagallar
}

DOI: http://dx.doi.org/10.17981/cultedusoc.9.3.2018.54

\author{
Artículo de investigación. Fecha de recepción: 15/06/2018. Fecha de aceptación: 27/11/2018 \\ Ana Sánchez Gómez²; \\ Luis Cabarcas Sánchez; Karina De Ayos González; \\ M. Rodríguez Galindo y Andrea Rangel Meriño ${ }^{3}$ \\ IED Rural de Cantagallar (Colombia) \\ cabarcasluis@gmail.com
}

Para citar este artículo:

Sánchez, A.,Cabarcas, L., De Ayos. K., Rodríguez, M. y Rangel, A. (2018). La investigación como estrategia para fomentar valores entre estudiantes de noveno grado de la institución educativa departamental rural de Cantagallar apoyada en las TIC. Cultura. Educación y Sociedad 9(3), 465-472. DOI: http://dx.doi.org/10.17981/cultedusoc.9.3.2018.54

\section{Resumen}

La puesta en práctica de los valores, ha resultado desde siempre imprescindible en la formación de los estudiantes y la adquisición de competencias ciudadanas integrales, por tal razón la presente investigación tiene como objetivo fomentar los valores, especialmente el respeto y la tolerancia en los estudiantes a través de la investigación como estrategia pedagógica. La metodología utilizada en el estudio es de tipo cualitativa, de alcance descriptivo, el diseño es soportado desde las cualidades de la IEP. Los instrumentos aplicados fueron la entrevista semiestructurada, observación participante la cual fue registrada a través de un diario de campo. Los participantes fueron 50 estudiantes de noveno grado de la institución educativa Departamental Rural de Cantagallar. Por lo tanto la ejecución de este proyecto permitió no solo el reconocimiento de los aspectos significativos, positivos y valorativos de los estudiantes, sino que además logró que se identificaran las falencias a nivel de convivencia, relaciones, manejo de conflictos, cohesión grupal, relación estudiante-docente y se participara en actividades que fortalecieran la armonía.

Palabras clave: estrategia, pedagógica, respeto, tolerancia.

\section{Abstract}

The implementation of values, has always been essential in the training of students and the acquisition of comprehensive citizenship skills, for this reason this research aims to promote values, especially respect and tolerance in students to through research as a pedagogical strategy. The methodology used in the study is qualitative, descriptive in scope, the design is supported from the qualities of the IEP. The instruments applied were the semi-structured interview, participant observation which was recorded through a field diary. The participants were 50 students of ninth grade of the educational institution of Rural Departmental of Cantagallar. Therefore the execution of this project allowed not only the recognition of the significant, positive and valuable aspects of the students, but also managed to identify the shortcomings at the level of coexistence, relationships, conflict management, group cohesion, student relationship-docente and participate in activities that strengthen harmony.

Keywords: strategy, pedagogical, respect, tolerance.

1 Este artículo ha sido derivado del Programa de Fortalecimiento de la Cultura Ciudadana y Democrática CT+I a través de la IEP apoyada en TIC en el Departamento de Magdalena: CICLON

2 Líder del Grupo de Investigación "Los Pacifistas", de la IED Rural de Cantagallar.

3 Docentes miembros del Grupo de Investigación "Los Pacifistas", de la IED Rural de Cantagallar

- The author; licensee Universidad de la Costa - CUC.

Cultura, Educación y Sociedad vol. 9 no. 3, pp. 465-472. Diciembre, 2018

Barranquilla. ISSN 2389-7724 Online 


\section{Introducción}

Es indudable que para garantizar una mayor calidad en la educación se hace necesario que los docentes alcancen un nivel de desarrollo profesional que les permita dar soluciones más efectivas a los problemas de su práctica pedagógica y esto se logra mediante la actividad investigativa, la cual de forma directa incentiva a una mayor capacidad de conocimiento (De armas, Lorences, Perdomo, 2003). Existen numerosas investigaciones en las que se demuestran que las representaciones de los alumnos son diferentes a los conceptos que se les quiere enseñar, por esto abrir un campo de ideas a los estudiantes para que desarrollen temas de su interés personal, es una forma de incentivar respeto y tolerancia, en lo que respecta la forma de pensar (Gagliardi, 1986).

La propuesta pedagógica consiste en impulsar actividades que conduzcan a generar acciones que permitan a los estudiantes adquirir ciertos comportamientos que impulsen la transformación en las relaciones interpersonales a través de valores como el respeto y la tolerancia como medio para garantizar una funcional y eficiente convivencia entre los miembros de una comunidad.

Sin embargo, este ideal que está en la base de cualquier sistema educacional debe ser confrontado con la realidad de las Instituciones y debe constituir sin duda, un desafío y responsabilidad de los saberes pedagógicos y disciplinarios.

Universidades de alto prestigio como la Universidad de Caldas, a través del departamento de estudios educativos como unidad formadora de docentes, en el contexto del sistema de acreditación y en la búsqueda de la calidad de la educación, considera al tema de la investigación inherente a la acción educativa. Pues la investigación de distintas formas resulta ser una estrategia de calidad para el desarrollo y formación de establecimientos educativos considerados de calidad (Quintero, Munevar, 2008). en la sociedad del conocimiento, se consideran entidades con alta calidad en la educación aquellas que se encuentran más asociadas al campo de la investigación, dicha investigación se puede manifestar de dos formas, una puede ser el enseñar a educar, y la otra el hacer dicha investigación (Restrepo, 2003).

La investigación puede ser vista desde la pedagogía y desde el ejercicio misional de generar conocimiento, propio de la educación superior. Desde los ámbitos vinculados a las prácticas escolares, los docentes y sus organizaciones colectivas vienen planteando serios cuestionamientos sobre las formas que han adoptado históricamente el diseño, el desarrollo y los productos de la investigación educativa, muchas instituciones solo toman el tema de investigación como un tema adicional a los ya establecidos, pero hay que tener en cuenta que cada actividad relacionada con el desarrollo intelectual, la investigación debe ser y el principal componente (Suarez, 2007).

Las implicaciones epistemológicas y pedagógicas que entraña el vínculo docenciainvestigación en el que hacer académico de las instituciones educativas de enseñanza media superior y superior, está muy relacionado con la idea de que los alumnos desarrollen habilidades de lectura y aprendizaje, y esto a su vez ayude a fomentar valores que de una u otra manera están relacionados con la intelectualidad del estudiante, un alumno educado e investigativo, entra dentro del perfil de un estudiante con valores y principios (Moran, 1993).

A modo de ejemplo, si bien es posible afirmar que la función de la investigación corresponde propiamente a los profesores, y que es de esta de donde deben alimentar la docencia, no siempre la función docente coincide con el ámbito de interés de investigación del profesor, es aquí donde el papel 
de los estudiantes toma mayor empoderamiento, y es que hablar de investigación no hace referencia siempre a personal experto en el tema, sino también a personas curiosas e interesadas en resolver alguna idea (Parra, 2004). La estrategia de aprender a aprender se presenta como alternativa para enfrentar muchos de los retos de la escuela del futuro. La estrategia del saber, poder y querer en el concepto de aprendizaje conforma un paisaje complejo e inacabado, vivo y apasionante que anima el debate entre los estudiosos (Pozo, 2001).

La investigación acción en la actualidad, resulta muy útil para tratar los análisis de nuevos desarrollos curriculares y tendencias metodológicas en la investigación educativa. Desarrollar temas de interés por un cierto periodo de tiempo, requiere de compromisos y tiempo, en muchas instituciones, se utiliza como herramienta de aprendizaje y desarrollo de valores y competencias (Pegudo, Cabrera, López, Cruz, 2012). Los estudiantes entre mayor nivel alcanzan en la educación, más conflictivos pueden llegar a ser, por esto, mantener sus mentes y espacios ocupados con estrategias de aprendizaje resulta de gran interés en las instituciones. La didáctica centrada en el estudiante exige la utilización de estrategias y métodos adecuados, en los que el aprendizaje se conciba cada vez más como resultado del vínculo entre lo afectivo, lo cognitivo, las interacciones sociales y la comunicación (Montes, Machado, 2011). La docencia actual, a pesar de los avances de la investigación educativa y de los programas de formación de profesores de los últimos años, con demasiada frecuencia se ha convertido en una actividad mecánica, improvisada y fría, de manera que la labor se lleva a cabo solo por ser cumplida y en muchas ocasiones no se preocupa por dejar huellas que realmente marquen el desarrollo de la formación de los estudiantes (Moran, 2004).

\section{La investigación como estrategia pedagógica}

La investigación es una empresa de conocimiento centrada en resolver problemas de conocimiento. Pero, por otra parte, el conocimiento pedagógico no se justifica sin el compromiso de mejorar la propia realidad que interpreta (Orden, 2007).

Enseñar con eficacia es uno de los problemas más relevantes en educación, la calidad de la práctica docente, va a depender de las estrategias que utilice el docente para llevar a cabo la realización de su clase, las excesivas actividades de planificación, y el poco tiempo para llevar a cabo la realización de proyectos, evitan que el educador genere procesos de investigación (Aranguren, 2007).

"Los Semilleros de Investigación, son en Colombia son una estrategia pedagógica extracurricular que tiene como finalidad fomentar la cultura investigativa en estudiantes del pregrado que se agrupan para desarrollar actividades que propendan por la formación investigativa, la investigación formativa y el trabajo en red" (COLCIENCIAS, 2006).

A los largo del tiempo, diversos estudios se han centrado en la idea de que las investigaciones, serian una buena herramienta pedagógica de aprendizaje, de restablecimiento y transformación de un individuo. Por muy indisciplinado que sea un estudiante, si se involucra en procesos de investigación, de manera que el estudiante se centre en temas de su interés, muy posiblemente su conducta se ira transformando y moldeando a medida que este se va comprometiendo con su labor.

La enseñanza de la investigación es una práctica cuyo proyecto es "exponer los principios de una práctica profesional y simultáneamente imprimir cierta relación a esa práctica, es decir proporcionar a la vez los instrumentos indispensables para el tratamiento sociológico del objeto y una disposición activa a utilizarlos" (Merlinsky, 2006). 
El carácter educativo que tiene la investigación, le da su propia identidad, debido a que la investigación por si sola contribuye a ayudar a comprender la evolución de la educación, identificando sus principales problemas, áreas de oportunidades y posibles perspectivas de desarrollo (Calixto, 2012).

\section{Valores: el respeto y la tolerancia.}

Los valores son aquellos principios y cualidades humanas que, unidos a una serie de características del comportamiento humano, como creencias, moral o ética, permiten a las personas ser mejores cada día más, además brindan la posibilidad de elegir entre diferentes situaciones, con la finalidad de elegir la más conveniente y la mejor (Frondizi, 1958). Conocer las estrategias cognitivas y metacognitivas que utilizan en situaciones de aprendizaje los estudiantes para desarrollar temas de su interés, es de vital importancia al momento de desear entender su forma de aprender y desarrollarse en el ámbito escolar (Correa, Castro, 2004).

La investigación es reconocida como un recurso básico, para la transformación de las prácticas pedagógicas, y el replanteamiento de las relaciones entre el conocimiento, la sociedad y el punto de partida hacia la conjugación de esfuerzos que articulen los desarrollos de la ciencia. Por otra parte, se asume que la generación de un pensamiento reflexivo y autónomo que oriente los procesos colectivos de construcción conceptual, permite superar y favorecer la transformación del pensamiento rutinario y espontáneo ( Callejas, Corredor, 2002).

La relación directa que tiene las investigación con los valores que se vean reflejados en una persona, se basan en la transformación que logra el mantener a una persona pensando en cosas productivas por algunas horas del día, el ser investigador o de alguna forma interesarse por esta, desarrolla comportamientos distintos en una persona, la forma de ver la vida y amoldarse a ella es vista desde otra perspectiva. El preferir tomar un libro y documentarse en él, sería una forma de reemplazo de actitudes vacías.

Un maestro ideal que desempeñe su labor en los inicios del siglo XXI debe ser aquel que promueva un proceso de enseñanza y aprendizaje desarrollador, de manera que conduzca al tránsito continuo hacia niveles superiores de desarrollo, con la finalidad de formar personalidades integrales y auto determinadas, capaces de transformarse en un contexto histórico (Linares, Cruz, 2013).

En la actualidad el bajo rendimiento académico de los estudiantes, se sigue considerando como una de las principales problemáticas que afecta tanto al estudiante como a la misma institución, y se cree que esto se debe a la falta de estrategias de aprendizaje y evaluación por parte de los docentes, pues en ocasiones se prefiere el simple hecho de dictar una clase para cumplir con su labor, en vez de pensar en posibles alternativas de desarrollo de la clase, la investigación es aquí donde toma su lugar como estrategia pedagógica para incentivar conocimiento y valores en los estudiantes (Cardozo, 2011).

\section{Metodología}

Se realizó un estudio de tipo cualitativo, pues esta busca conocer e interpretar la realidad de los participantes a través de sus propias experiencias, entregando una información subjetiva del fenómeno de estudio. (Hernández, Fernández, y Baptista, 2010). El alcance de la investigación es descriptiva y con un diseño desde las cualidades específicas de la IEP reflexión y sistematización de experiencias. 


\section{Técnica e instrumentos de recolección de la información}

Las técnicas para la recolección de la información fueron la entrevista semiestructurada, la observación participante, empleando el diario de campo y guion de entrevista.

\section{Escenario y actores}

La población participante fueron 50 estudiantes de noveno grado de la institución educativa Departamental Rural de Cantagallar. Para la escogencia de los actores se realizó un diagnostico en donde hubiese recurrencia de problemas académicos y de convivencia por la ausencia de valores como el respeto y la tolerancia.

\section{Procedimiento}

El procedimiento del proyecto de investigación se realizó a partir de los recorridos de las trayectorias de indagación, basados en la integración de la IEP apoyada en TIC al aula, articulado al plan de estudios, a todas las áreas del conocimiento, lo anterior con la finalidad de generar una trasformación social y educativa a través del aprendizaje en contexto, involucrando intereses, motivaciones y rea- lidades de los estudiantes para generar conocimiento científico, donde se establecieron cinco (5) trayectos que especifican la ejecución y las metas trazadas para darle solución a la pregunta problema planteada. Los trayectos utilizados se presentan a continuación:

Trayecto (1): Se realizó un diagnóstico, de acuerdo a información observada y suministrada por los maestros frente a las acciones y comportamientos de los estudiantes, la identificación y promulgación de valores con el fin de identificar las dificultades específicas que presentaba la población.

Trayecto (2): Diseño de estrategias basadas en la investigación como estrategia pedagógica que fomenten los valores específicamente el respeto y la tolerancia y que puedan articularse de acuerdo a las necesidades de la población.

Trayecto (3): Implementación y transversalización de las estrategias en el aula de clase por parte del cuerpo docente.

Trayecto (4): análisis e interpretación de los resultados.

Trayecto (5): Reflexión y apropiación social sobre los conocimientos generados en materia de estrategias pedagógicas orientadas al aprendizaje y fortalecimiento.

\begin{tabular}{|c|c|c|}
\hline $\begin{array}{l}\text { Estrategia } \\
\text { Pedagogica }\end{array}$ & Actividad & Logros Alcanzados \\
\hline $\begin{array}{l}\text { Reconocimiento } \\
\text { de habilidades }\end{array}$ & $\begin{array}{l}\text { Inicialmente se trabaja identificando } \\
\text { las habilidades de los estudiantes, con } \\
\text { el fin de saber qué es lo que más le } \\
\text { gusta hacer, como hacen y que se les } \\
\text { dificultan resultando un aspecto por } \\
\text { mejorar. }\end{array}$ & $\begin{array}{l}\text { Los estudiantes se mostraron } \\
\text { participativos, y aunque no sabían que } \\
\text { en las actividades se estaba evaluando } \\
\text { y reconociendo las capacidades y } \\
\text { habilidades lograron asumir con } \\
\text { responsabilidad las funciones } \\
\text { asignadas para el cumplimiento final } \\
\text { de las tareas. }\end{array}$ \\
\hline
\end{tabular}




\begin{tabular}{|c|c|c|}
\hline $\begin{array}{l}\text { Estrategia } \\
\text { Pedagogica }\end{array}$ & Actividad & Logros Alcanzados \\
\hline
\end{tabular}

Es importante observar los roles que los estudiantes asumen en el aula, pues esto va de la mano con las capacidades de cada uno y la manera en la que ponen en práctica diferentes

Roles identificables

Valores integrales

Articulación áreas de estudio

Estudiantes y Docentes: un mismo valor acciones que pueden ir a favor o en contra de la convivencia, por lo tanto, es en la identificación compromiso que se denota la existencia de valores y la promoción de los mismo en cada una de las actividades. En esta actividad se realizó simulación de caso cotidiano presentado en el salón de clases.

Los valores integrales permiten definir el accionar de los individuos. La actividad se realizó a través de dramatizados en donde se deberían promover los valores, especialmente el respeto y la tolerancia y cómo estos eran importante en cada clase.

En apoyo con los docentes se realiza una socialización pedagógica con los estudiantes en donde se ejemplifica la importancia de los valores en todas las ar4eas de estudio, y como los docentes las pueden implementar logrando la participación de todos.

A través de un Roll Playing se realiza una actividad en donde los docentes asumen el papel de estudiantes y viceversa, logrando mostrar cuáles son las situaciones que debilitan la puesta en práctica de valores, y como se maneja los conflictos.
Los estudiantes reconocen que ante los temas que se desarrollan en el salón de clases cada quien asume un rol diferente, algunos positivos y otros negativos que son desencadenantes le un mal clima, y por ende se pudo notar los detonantes de la convivencia y las relaciones con los compañeros, a lo cual se comprometen a participar activamente con el fin de erradicarlos.

Asumen la importancia de los valores en su formación académica, así mismo ven la necesidad de que sean los maestros los que de acuerdo a su metodología de trabajo los tengan en cuenta en cada actividad que se realice en el aula.

Dicen que los valores están presente de todo lo que el maestro explica, y además en la convivencia que se tiene co los compañeros, por lo tanto están dispuestos a participar y les motiva la idea de que en cada asignatura ofertada existan temas de valores, pues de eso depende convertirse en un ciudadano de bien.

Los estudiantes y docentes saben que el fortalecimiento de la puesta en práctica de valores como el respeto y la tolerancia se consigue si se trabaja en conjunto y todos actúan de acuerdo a los mismos propósitos, por tal motivo les gusta mucho la idea de que los docentes sean unos estudiantes más al momento de realizar estas actividades.

\section{Resultados}

Entre los hallazgos podemos destacar que posterior a la intervención en los estudiantes, se podía observar como estos desarrollaban habilidades y tenían menos inconvenientes con las personas a su alrededor. Los alumnos de noveno grado mostraron resultados positivos y cada día se veían más interesados por el tema de investigación elegido por ellos mismos, de manera que se espera que para un futuro la población de estudiantes conflictivos en la institución Educativa Departamental Rural de Cantagallar haya disminuido significativamente. 


\section{Conclusión}

La investigación como estrategia de investigación, resulta ser muy útil al momento de querer fomentar en los estudiantes mejores principios y costumbres, pues esta contribuye de manera directa al desarrollo intelectual, mantiene a los estudiantes ocupados en cosas productivas y logra una transformación positiva en el estudiante, de manera que si se trata de esta forma, en un futuro se podría hablar de estudiantes más intelectuales y de mucho más compromiso.

Por lo tanto la ejecución de este proyecto permitió no solo el reconocimiento de los aspectos significativos, positivos y valorativos de los estudiantes, sino que además logró que se identificaran las falencias a nivel de convivencia, relaciones, manejo de conflictos, cohesión grupal, relación estudiante-docente y se participara en actividades que fortalecieran la armonía.

Cuando se habla del fomento de valores en los estudiantes en un aula de clases no se puede olvidar que quienes lideran el proceso son los maestros, por tal razón son ellos los que deben estar mayormente sintonizados con los procesos pedagógicos, en especial con el tema de los valores ellos deben articular desde lo que dicen los planes de estudio todas las tareas logrando que la temática aborde el objetivo final.

Aunque los resultados fueron positivos, y los estudiantes lograron mejorar sus relaciones convivenciales, y llevan al ejercicio práctico acciones que fomenten valores, se debe seguir trabajando arduamente en el tema, e incluir a los padres de familia como primera institución de formación en valores, al igual que seguir diseñando estrategias docentes para llevarlas al aula.

\section{Referencias}

Calixto, R. (2012). Investigación en educación ambiental. Revista mexicana de investigación educativa, 17(55), 1019-1033.

Callejas, M. y Corredor, M. (2002). La renovación de los estilos pedagógicos: colectivos para la investigación y la acción en la universidad. Revista docencia universitaria, 3(1).

Cardozo-Ortiz, C. (2011). Tutoría entre pares como una estrategia pedagógica universitaria. Educación y Educadores, 14(2).

Correa, M., Castro, F. y Lira, H. (2004). Estudio descriptivo de las estrategias cognitivas y metacognitivas de los alumnos y alumnas de primer año de pedagogía en enseñanza media de la Universidad del Bío-Bío. Theoria, 13(1). 103-110.

De Armas, N., Lorences, J. y Perdomo, J. (2003). Caracterización y diseño de los resultados científicos como aportes de la investigación educativa. Evento Internacional Pedagogía, 40. Recuperado de http://files.especializacion-2012. webnode.com.co/200000152. $80 \mathrm{~d} 3 \mathrm{f} 81 \mathrm{ccc} / \mathrm{CAR}$ ACTERIZACIÓN\%20Y\%20DISEÑO\%20DE\%20 LOS\%20RESULTADOS\%20CIENTÍFICOS\%20COMO \% 20APORT E S \% $20 \mathrm{DE} \% 20 \mathrm{~L} \mathrm{~A} \mathrm{\%} 20$ INVESTIGACIÓN\%20EDUCATIVA..pdf

Frondizi, R. (1958). ¿Qué son los valores? México, D.F.: Fondo de cultura económica.

Gagliardi, R. (1986). Los conceptos estructurales en el aprendizaje por investigación. Enseñanza de las ciencias: revista de investigación y experiencias didácticas, 4(1), 30-35. 
González, J. (2008). Semilleros de Investigación: una estrategia formativa. Psychologia. Avances de la disciplina, 2(2), 185-190.

Linares, M. y Cruz, D. (2013). Estrategia de superación pedagógica para docentes de la carrera de Medicina. Educación Médica Superior, 27(4), 340-355.

Quintero-Corzo, J., Munévar-Molina, R. A. y Munévar-Quintero, F. (2008). Semilleros de investigación: una estrategia para la formación de investigadores. Educación y educadores, 11(1). 31-42.

Merlinsky, G. (2006). La entrevista como forma de conocimiento y como texto negociado: notas para una pedagogía de la investigación. Cinta de moebio, (27).

Morán, P. (2004). La docencia como recreación y construcción del conocimientoSentidopedagógico delainvestigación en el aula. Perfiles educativos, 26(105106), 41-72.
Morán, P. (1993). La vinculación docencia investigación como estrategia pedagógica. Perfiles educativos, (61).

Montes de Oca, N. y Machado, E. (2011). Estrategias docentes y métodos de enseñanza-aprendizaje en la Educación Superior. Humanidades Médicas, 11(3), 475-488.

Parra, C. (2004). Apuntes sobre la investigación formativa. Educación y educadores, (7). 55-77.

Pegudo, A., Cabrera, M., López, E. y Cruz, L. (2012). Estrategia pedagógica para desarrollar habilidades investigativas en asesores de trabajo de investigación científico estudiantil. Edumecentro, 4(1), 95103.

Pozo, J. y Monereo, C. (2001). El aprendizaje estratégico. Docencia universitaria, 2(2).

Orden, A. (2007). El nuevo horizonte de la investigación pedagógica. Revista Electrónica de Investigación Educativa, 9(1), 1-22. 